\title{
Endoglin involvement in integrin-mediated cell adhesion as a putative pathogenic mechanism in hereditary hemorrhagic telangiectasia type 1 (HHT1)
}

\author{
Elisa Rossi ${ }^{1}$, José M. Lopez-Novoa ${ }^{2}$ and Carmelo Bernabeu ${ }^{3}$ * \\ 1 INSERM, Faculté des Sciences Pharmaceutiques et Biologiques, Université Paris Descartes, UMR-S 1140, Paris, France \\ 2 Renal and Cardiovascular Research Unit, Department of Physiology and Pharmacology, University of Salamanca, and Institute of Biomedical Research of \\ Salamanca, Salamanca, Spain \\ ${ }^{3}$ Centro de Investigaciones Biológicas - Consejo Superior de Investigaciones Científicas and Centro de Investigación Biomédica en Red de Enfermedades \\ Raras, Madrid, Spain
}

\section{Edited by:}

Michelle Letarte, University of Toronto and Hospital for Sick Children, Canada

\section{Reviewed by:}

Christina Stuelten, National Cancer

Institute, USA

Theodora Katsila, University of Patras, Greece

\section{*Correspondence:}

Carmelo Bernabeu, Centro de Investigaciones Biológicas - Consejo

Superior de Investigaciones

Científicas and Centro de

Investigación Biomédica en Red de Enfermedades Raras, Calle Ramiro de Maeztu 9, Madrid 28040, Spain e-mail: bernabeu.c@cib.csic.es
Mutations in the endoglin gene $(E N G)$ are responsible for $\sim 50 \%$ of all cases with hereditary hemorrhagic telangiectasia (HHT). Because of the absence of effective treatments for HHT symptoms, studies aimed at identifying novel biological functions of endoglin which could serve as therapeutic targets of the disease are needed. Endoglin is an endothelial membrane protein, whose most studied function has been its role as an auxiliary receptor in the TGF- $\beta$ receptor complex. However, several lines of evidence suggest the involvement of endoglin in TGF- $\beta$-independent functions. Endoglin displays, within its zona pellucida domain, an RGD motif, which is a prototypic sequence involved in integrinbased interactions with other proteins. Indeed, we have recently described a novel role for endothelial endoglin in leukocyte trafficking and extravasation via its interaction with leukocyte integrins. In addition, functional, as well as protein and gene expression analysis have shown that ectopic endoglin represses the synthesis of several members of the integrin family and modulates integrin-mediated cell adhesions. This review focuses on the tight link between endoglin and integrins and how the role of endothelial endoglin in integrin-dependent cell adhesion processes can provide a better understanding of the pathogenic mechanisms leading to vascular lesions in endoglin haploinsufficient HHT1 patients.

Keywords: HHT, endoglin, integrins, cell adhesion, endothelial cells, inflammation, RGD

\section{ENDOGLIN INVOLVEMENT IN HEREDITARY HEMORRHAGIC TELANGIECTASIA}

Hereditary hemorrhagic telangiectasia (HHT) or Rendu-OslerWeber syndrome is a vascular hereditary autosomal dominant disease associated with epistaxis, telangiectasias, gastrointestinal hemorrhages, and arteriovenous malformations in lung, liver, and brain (Shovlin, 2010; McDonald et al., 2011). The first gene identified as being involved in HHT was ENDOGLIN (ENG) that maps to chromosome 9 (Fernández-Ruiz et al., 1993; McAllister et al., 1994), representing between 39 and 59\% of the total HHT population, a frequency that depends on the geographic location. Several 100 different pathogenic mutations have been described in ENG giving rise to the HHT1 subtype (Abdalla and Letarte, 2006). Haploinsufficiency is accepted as the underlying mechanism of HHT1 pathogenicity, but the fine cellular and molecular machinery involved remains to be elucidated.

\section{ENDOGLIN STRUCTURE, EXPRESSION, AND FUNCTION}

Human endoglin is a type I integral membrane protein with a large extracellular (EC) domain (561 amino acids), a single hydrophobic transmembrane (TM) domain, and a short cytosolic tail (Figure 1A). Endoglin is a glycosylated protein expressed as a $180-\mathrm{kDa}$ disulfide-linked homodimer in endothelial cells (Gougos and Letarte, 1990). The predominant expression of endoglin in endothelial cells suggests that these are the target cells in HHT, where endoglin haploinsufficiency reveals its pathogenicity. Structurally, endoglin belongs to the zona pellucida (ZP) family of proteins that share a juxtamembrane ZP domain of 260 amino acid residues in their EC region (Llorca et al., 2007). This ZP domain encodes an RGD tripeptide that is a prototypic member of a family of motifs involved in integrinbased interactions with EC matrix and certain cell surface proteins (Takada etal., 2007). Recently, it has been demonstrated that endothelial endoglin interacts with leukocyte integrin $\alpha 5 \beta 1$ via its RGD motif, suggesting a regulatory role for endoglin in transendothelial leukocyte trafficking (Rossi et al., 2013). The RGD motif is present in endoglin from primates, while functionally related RGD-like motifs are found in endoglin from rodents and other mammals, stressing the physiological importance and high conservation of this motif (Gougos and Letarte, 1990; Rossi et al., 2013). Next to the ZP domain is the orphan domain, which is located in the NH2-terminus of the protein (Llorca et al., 2007). The orphan domain is involved in the binding to members of the TGF- $\beta$ superfamily (activin, TGF- $\beta$, and BMP families) in accordance with the role of endoglin as an auxiliary 


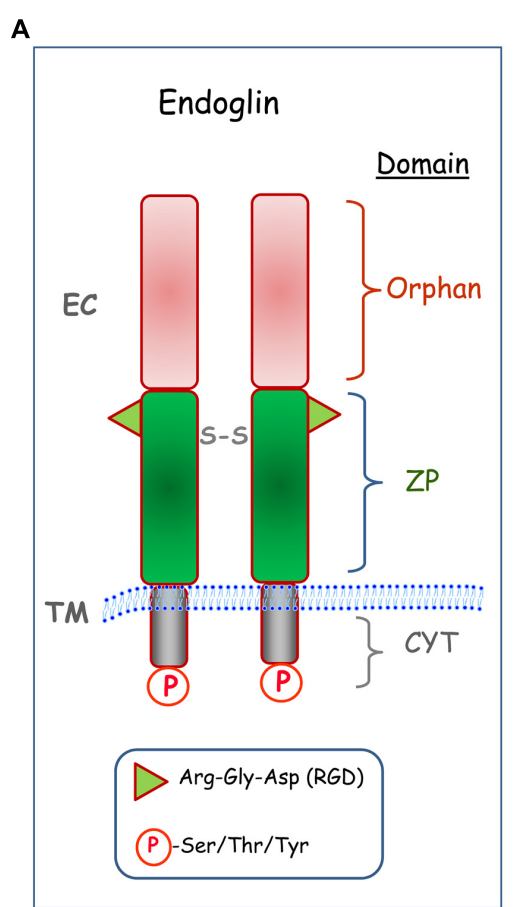

B

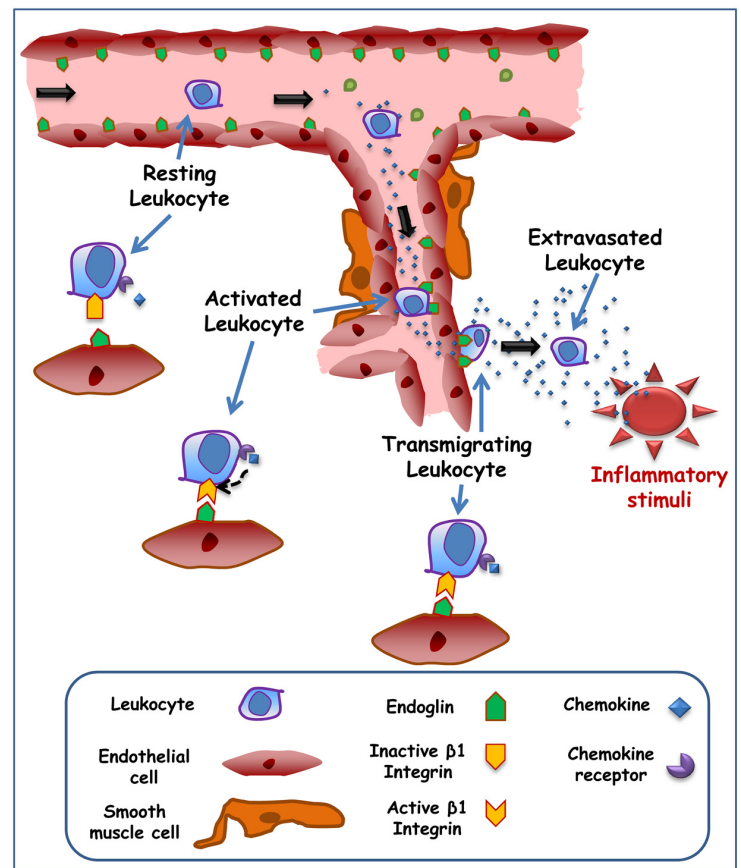

FIGURE 1 | Structure and cell adhesion function of endoglin. (A) Structural representation of endoglin. Endoglin is a type I membrane protein with a large extracellular (EC) domain that contains a zona pellucida (ZP) domain in the juxtamembrane region and an $\mathrm{NH}_{2}$-terminal orphan domain. The ZP domain encodes an Arg-Gly-Asp (RGD) tripeptide that is involved in integrin binding, whereas de orphan domain is involved in binding to members of the TGF- $\beta$ superfamily. Endoglin forms dimers and the corresponding monomers are disulphide linked (S-S). The cytoplasmic (CYT) domain can be phosphorylated (P) at Ser/Thr/Tyr residues. The transmembrane (TM), and EC domains of the protein are indicated. The scheme is not to scale. (B) Role of endothelial endoglin in leukocyte adhesion and transmigration. A schematic diagram shows a hypothetical model for leukocyte transmigration through the vessel endothelium. In an inflammatory focus, different soluble factors are released, including the chemokine CXCL12, leading to activation and endoglin-dependent extravasation of leukocytes. The transmigration process of the leukocyte involves the binding of CXCL12 to its receptor CXCR4, which in turn activates $\beta 1$ integrins. Once activated, $\beta 1$ integrin binds to the RGD motif of endoglin present on the endothelial cell surface, allowing the extravasation and migration of leukocytes to the inflammatory site.
TGF- $\beta$ receptor (Alt et al., 2012). Thus, endoglin modulates the endothelial response to TGF- $\beta$-related ligands, including cell proliferation, apoptosis, vascular remodeling, angiogenesis, wound healing, or fibrosis (López-Novoa and Bernabeu, 2010). Of note, most of ENG mutations associated with HHT1 map to the EC domain, suggesting that the biological function of this domain is critical for the disease (Bernabeu et al., 2010). The cytosolic domain of endoglin is phosphorylated at Ser/Thr/Tyr residues and it can be targeted by serine/threonine (Lastres et al., 1994) and tyrosine (Pan et al., 2014) kinases. It has been shown that the endoglin phosphorylation status can influence its subcellular localization (Koleva et al., 2006) and degradation (Pan et al., 2014). The cytoplasmic domain of endoglin also regulates actin cytoskeletal organization, microtubule-based transport machinery, and cell migration (Ray et al., 2010; Romero et al., 2010), through its specific binding to different cytosolic proteins (López-Novoa and Bernabeu, 2010).

\section{ENDOGLIN IN INTEGRIN-MEDIATED SIGNALING AND CELLULAR FUNCTION}

Integrins are ubiquitous cell surface receptors involved in cell-cell and cell-EC matrix interactions (Takada et al., 2007) that play a relevant role in vascular biology (Plow et al., 2014). The functional role of endothelial endoglin as a counter-receptor for leukocytes' integrins has been recently reported (Rossi etal., 2013). Interestingly, inflammatory leukocyte recruitment, which involves integrin-mediated cell-cell interactions, is critical for proper vascular remodeling. Several animal models of inflammation and vascular repair have shown that endoglin haploinsufficiency leads to an abnormal leukocyte infiltration and function that depends on the target organ or the triggering stimulus (van Laake et al., 2006; Jerkic et al., 2010; Rossi et al., 2013; Ardelean et al., 2014; Peter et al., 2014; Shen et al., 2014). Thus, an inflammation model of dextran sodium sulfate (DSS)-induced chronic colitis revealed more leukocyte infiltration in the gut and a more severe colitis phenotype in endoglin heterozygous $\left(\mathrm{Eng}^{+/-}\right)$mice relative to control animals (Jerkic et al., 2010; Ardelean et al., 2014; Peter et al., 2014). After myocardial infarction, a greater deterioration in cardiac function was observed in $\mathrm{Eng}^{+/-}$compared to control mice, although host inflammatory leukocyte numbers in the infarct zone were similar; however, defects in vessel formation and heart function in $\mathrm{Eng}^{+/-}$mice were rescued by injection of leukocytes from healthy human donors, but not by leukocytes from HHT1 patients (van Laake et al., 2006). Moreover, 
upon permanent distal middle cerebral artery occlusion, $E n g^{+/-}$ mice showed larger infarct/atrophic volumes associated with fewer infiltrating macrophages, suggesting that endoglin deficiency impairs brain injury recovery by impairing macrophage homing, delaying inflammation resolution, and reducing angiogenesis (Shen et al., 2014). Furthermore, decreased inflammationinduced leukocyte trafficking to peritoneum and lungs was found in Eng ${ }^{+/-}$mice treated with the inflammatory agents carrageenan or lipopolysaccharide (LPS), respectively (Rossi et al., 2013). The impaired leukocyte trafficking was observed for neutrophils, lymphocytes, monocytes, and basophiles. The nature of this migratory process was assessed using in vitro cell adhesion and transmigration studies. Leukocytes treated with the chemokine CXCL12, an integrin activator, strongly adhered to endoglin-coated plates and to endoglin-expressing endothelial cells. Similarly, leukocyte transmigration through adherent cell monolayers was dependent on the presence of endoglin. Both, endoglin-dependent cellular adhesion and transmigration processes involved the leukocyte integrin $\alpha 5 \beta 1$ via the endoglin RGD motif (Rossi et al., 2013). Based on these results, a hypothetical model is illustrated in Figure 1B.

Additional lines of evidence support a tight link between integrins and endoglin functions: (i) on overexpression of endoglin, fibroblasts tend to form clusters that involve $\alpha 5 \beta 1$ integrin and are inhibited by coating the culture plates with an RGD-containing fragment of fibronectin (Guerrero-Esteo et al., 1999); (ii) analysis of integrin-rich sites of focal adhesion, isolated with RGD-labeled microspheres, shows that endoglin regulates cell migration and focal adhesion composition via interaction through its cytoplasmic domain with the protein zyxin present in focal adhesion sites (Conley et al., 2004); (iii) binding of human pathogenic bacteria to the carcinoembryonic antigen (CEA) in epithelial cells, triggers de novo expression of endoglin, which, in turn changes focal adhesion composition, activating $\beta 1$ integrins and inducing a dramatic increase in the ECM-binding capacity of the cells (Muenzner et al., 2010); (iv) endoglin mediates fibronectin $/ \alpha 5 \beta 1$ integrin and TGF- $\beta$ pathway crosstalk in endothelial cells via the internalization of the cell surface $\alpha 5 \beta 1$ integrin/endoglin complex (Tian et al., 2012); and (v) protein and gene expression analysis have shown that ectopic endoglin represses the synthesis of several members of the integrin family and modulates integrin-related biological functions, including cell adhesion and transmigration (Aristorena et al., 2014; Blanco et al., 2014). Moreover, endoglin is able to antagonize the integrins' downstream MAPK pathway, by inhibiting ERK signaling and altering the subcellular distribution of activated ERK (Lee and Blobe, 2007; Santibanez et al., 2010). Taken together, these data strongly suggest a close physical and functional association between endoglin and integrins and prompt the question of whether the adhesion role of endothelial endoglin, as a counter-receptor for leukocytes' integrins, is involved in the HHT pathogenesis.

\section{IS THE ROLE OF ENDOGLIN IN CELL-CELL ADHESION INVOLVED IN HHT PATHOGENESIS?}

A common feature in HHT patients is the presence of vascular lesions (telangiectasias and arteriovenous malformations) that lead to a loss of the intervening capillary network that connects the arteriole with the venule (Braverman etal., 1990). However, it remains unclear why the vascular lesions appear only at distinct sites within certain organs, rather than being present throughout the body and in all organs/tissues. To explain this paradox, the need for a trigger such as inflammation, vascular injury, angiogenic stimuli, or ischemia has been postulated (Park et al., 2009; López-Novoa and Bernabeu, 2010; Choi et al., 2014). In HHT1, this trigger would synergize with endoglin haploinsufficiency to generate the vascular lesion (Lopez-Novoa, 2012; Choi etal., 2014). Interestingly, those stimuli are usually associated with the upregulated expression of endoglin in endothelial cells and an inflammatory cell infiltrate (Torsney et al., 2002; López-Novoa and Bernabeu, 2010), suggesting the need of both, endoglin function and leukocyte infiltration, in the vascular repair/remodeling process. Indeed, it has been reported that endoglin plays a crucial role in leukocyte-mediated vascular repair (van Laake etal., 2006). Of note, HHT skin telangiectasias show a perivascular mononuclear cell infiltrate, including lymphocytes and monocytes/macrophages (Braverman etal., 1990). This is in agreement with the leukocyte infiltration occurring during angiogenesis and vascular remodeling (Jaipersad etal., 2014). Therefore, it can be hypothesized that the function of endothelial endoglin as an adhesion counterreceptor for leukocyte's integrins is involved in HHT1 pathogenesis (Figure 2). According to this hypothetical model, in healthy

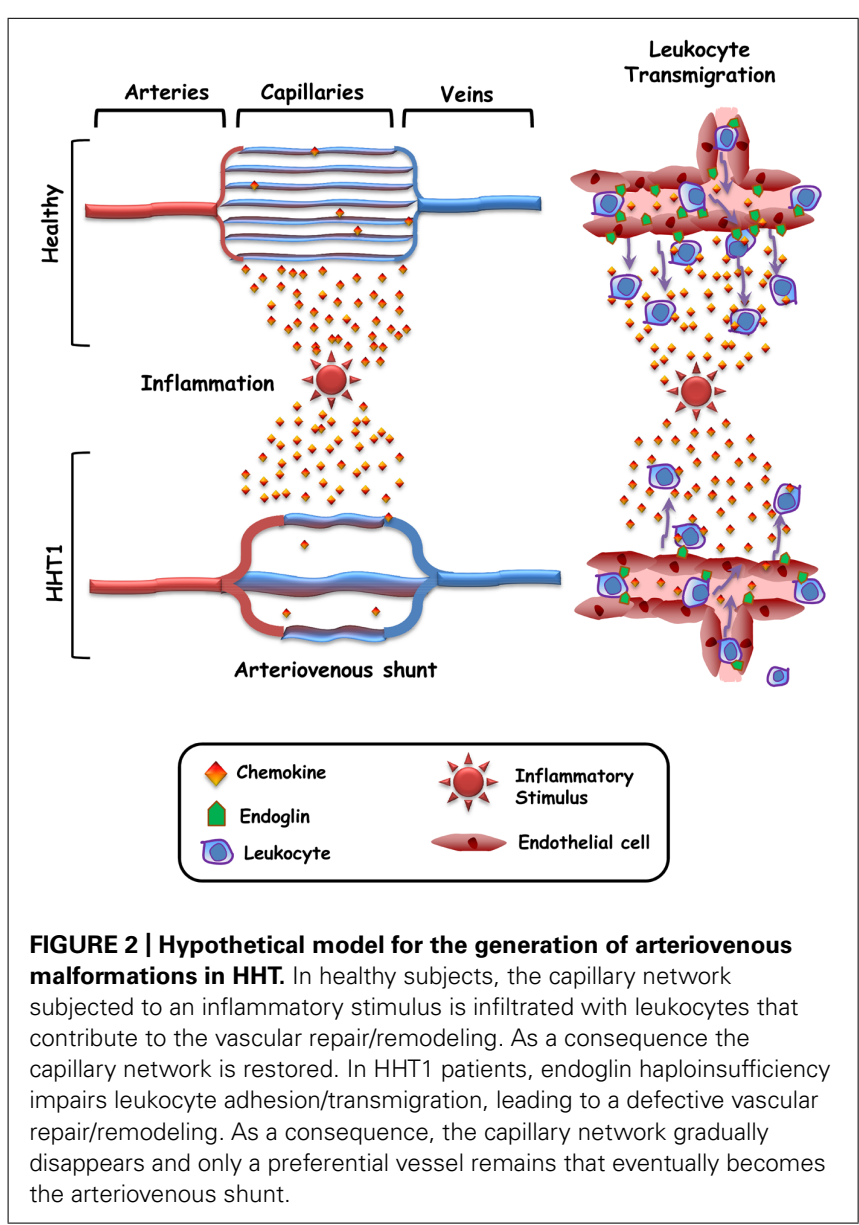


subjects the capillary network subjected to an inflammatory stimulus is infiltrated with leukocytes that contribute to the vascular repair/remodeling. By contrast, in HHT1 patients endoglin haploinsufficiency impairs leukocyte infiltration, leading to a defective vascular repair/remodeling. As a consequence, the capillary network would disappear and only a preferential vessel remains that eventually becomes the arterio-venous shunt.

\section{CONCLUSION AND PERSPECTIVES}

A novel role for endothelial endoglin as a cellular adhesion receptor has been reported. Here, we postulate a model attempting to relate the cell adhesion function of endoglin to the pathobiology of HHT. In the HHT1 setting, endoglin protein levels may not reach the minimum threshold to achieve the optimal function in leukocyte adhesion mediated by integrins. This endoglin haploinsufficiency alters leukocyte transmigration and may synergize with a trigger such as inflammation to generate the vascular lesion. The function of endoglin as an adhesion counter-receptor for integrins may help to understand the pathogenicity in HHT and may be a potential therapeutic target in HHT. Modulators of endoglin/integrin cell adhesion are putative drugs to be tested. In addition to the endothelial cell-leukocyte adhesion, it would be of interest to investigate whether the adhesion of endothelial cells with mural cells also involves the endoglin/integrin interaction.

\section{ACKNOWLEDGMENTS}

This study was supported by grants from the Ministerio de Economiay Competitividad of Spain (SAF2010-19222 and SAF2013-43421-R to Carmelo Bernabeu; SAF2010-1588, SAF2013-45784-R, and RD12/0021/0032-REDINREN to José M. Lopez-Novoa). The Centro de Investigación Biomédica en Red de Enfermedades Raras (CIBERER) and REDINREN are initiatives of the Instituto de Salud Carlos III (ISCIII) of Spain supported by FEDER funds.

\section{REFERENCES}

Abdalla, S. A., and Letarte, M. (2006). Hereditary haemorrhagic telangiectasia: current views on genetics and mechanisms of disease. J. Med. Genet. 43, 97-110. doi: 10.1136/jmg.2005.030833

Alt, A., Miguel-Romero, L., Donderis, J., Aristorena, M., Blanco, F. J., Round, A., et al. (2012). Structural and functional insights into endoglin ligand recognition and binding. PLoS ONE 7:e29948. doi: 10.1371/journal.pone.0029948

Ardelean, D. S., Yin, M., Jerkic, M., Peter, M., Ngan, B., Kerbel, R. S., et al. (2014). Anti-VEGF therapy reduces intestinal inflammation in Endoglin heterozygous mice subjected to experimental colitis. Angiogenesis 17, 641-659. doi: 10.1007/s10456-014-9421-x

Aristorena, M., Blanco, F. J., de Las Casas-Engel, M., Ojeda-Fernandez, L., GallardoVara, E., Corbi, A., et al. (2014). Expression of endoglin isoforms in the myeloid lineage and their role during aging and macrophage polarization. J. Cell Sci. 127, 2723-2735. doi: 10.1242/jcs.143644

Bernabeu, C., Blanco, F. J., Langa, C., Garrido-Martin, E. M., and Botella, L. M. (2010). Involvement of the TGF-beta superfamily signalling pathway in hereditary haemorrhagic telangiectasia. J. Appl. Biomed. 8, 169-177. doi: 10.2478/v10136009-0020-x

Blanco, F. J., Ojeda-Fernandez, L., Aristorena, M., Gallardo-Vara, E., Benguria, A., Dopazo, A., et al. (2014). Genome-wide transcriptional and functional analysis of endoglin isoforms in the human promonocytic cell line U937. J. Cell. Physiol. doi: 10.1002/jcp.24827 [Epub ahead of print].

Braverman, I. M., Keh, A., and Jacobson, B. S. (1990). Ultrastructure and three-dimensional organization of the telangiectasias of hereditary hemorrhagic telangiectasia. J. Invest. Dermatol. 95, 422-427. doi: 10.1111/1523-1747.ep125 55569

Choi, E. J., Chen, W., Jun, K., Arthur, H. M., Young, W. L., and Su, H. (2014). Novel brain arteriovenous malformation mouse models for type 1 hereditary hemorrhagic telangiectasia. PLOS ONE 9:e88511. doi: 10.1371/journal.pone. 0088511

Conley, B. A., Koleva, R., Smith, J. D., Kacer, D., Zhang, D., Bernabéu, C., et al. (2004). Endoglin controls cell migration and composition of focal adhesions: function of the cytosolic domain. J. Biol. Chem. 279, 27440-27449. doi: 10.1074/jbc.M312561200

Fernández-Ruiz, E., St-Jacques, S., Bellón, T., Letarte, M., and Bernabéu, C. (1993). Assignment of the human endoglin gene (END) to $9 \mathrm{q} 34 \longrightarrow$ qter. Cytogenet. Cell Genet. 64, 204-207. doi: 10.1159/000133576

Gougos, A., and Letarte, M. (1990). Primary structure of endoglin, an RGDcontaining glycoprotein of human endothelial cells. J. Biol. Chem. 265, 8361-8364.

Guerrero-Esteo, M., Lastres, P., Letamendía, A., Pérez-Alvarez, M. J., Langa, C., López, L. A., et al. (1999). Endoglin overexpression modulates cellular morphology, migration, and adhesion of mouse fibroblasts. Eur. J. Cell Biol. 78, 614-623. doi: 10.1016/S0171-9335(99)80046-6

Jaipersad, A. S., Lip, G. Y., Silverman, S., and Shantsila, E. (2014). The role of monocytes in angiogenesis and atherosclerosis. J. Am. Coll. Cardiol. 63, 1-11. doi: 10.1016/j.jacc.2013.09.019

Jerkic, M., Peter, M., Ardelean, D., Fine, M., Konerding, M. A., and Letarte, M. (2010). Dextran sulfate sodium leads to chronic colitis and pathological angiogenesis in Endoglin heterozygous mice. Inflamm. Bowel Dis. 16, 1859-1870. doi: $10.1002 /$ ibd. 21288

Koleva, R. I., Conley, B. A., Romero, D., Riley, K. S., Marto, J. A., Lux, A., et al. (2006). Endoglin structure and function: determinants of endoglin phosphorylation by transforming growth factor-beta receptors. J. Biol. Chem. 281, 25110-25123. doi: 10.1074/jbc.M601288200

Lastres, P., Martín-Perez, J., Langa, C., and Bernabéu, C. (1994). Phosphorylation of the human-transforming-growth-factor-beta-binding protein endoglin. Biochem. J. 301, 765-768.

Lee, N. Y., and Blobe, G. C. (2007). The interaction of endoglin with betaarrestin2 regulates transforming growth factor-beta-mediated ERK activation and migration in endothelial cells. J. Biol. Chem. 282, 21507-21517. doi: 10.1074/jbc.M700176200

Llorca, O., Trujillo, A., Blanco, F. J., and Bernabeu, C. (2007). Structural model of human endoglin, a transmembrane receptor responsible for hereditary hemorrhagic telangiectasia. J. Mol. Biol. 365, 694-705. doi: 10.1016/j.jmb.2006. 10.015

Lopez-Novoa, J. M. (2012). Angiogenic stimuli and endoglin absence induces brain arteriovenous malformations: are local endoglin deletion and angiogenesis the 'second hit' that is necessary for arteriovenous malformations formation in HHT1? Cerebrovasc. Dis. 33:548. doi: 10.1159/000338772

López-Novoa, J. M., and Bernabeu, C. (2010). The physiological role of endoglin in the cardiovascular system. Am. J. Physiol. Heart Circ. Physiol. 299, H959-H974. doi: 10.1152/ajpheart.01251.2009

McAllister, K. A., Grogg, K. M., Johnson, D. W., Gallione, C. J., Baldwin, M. A., Jackson, C. E., et al. (1994). Endoglin, a TGF-beta binding protein of endothelial cells, is the gene for hereditary haemorrhagic telangiectasia type 1. Nat. Genet. 8, 345-351. doi: 10.1038/ng1294-345

McDonald, J., Bayrak-Toydemir, P., and Pyeritz, R. E. (2011). Hereditary hemorrhagic telangiectasia: an overview of diagnosis, management, and pathogenesis. Genet. Med. 13, 607-616. doi: 10.1097/GIM.0b013e3182 $136 \mathrm{~d} 32$

Muenzner, P., Bachmann, V., Zimmermann, W., Hentschel, J., and Hauck, C. R. (2010). Human-restricted bacterial pathogens block shedding of epithelial cells by stimulating integrin activation. Science 329, 1197-1201. doi: 10.1126/science.1190892

Pan, C. C., Kumar, S., Shah, N., Hoyt, D. G., Hawinkels, L. J., Mythreye, K., et al. (2014). Src-mediated post-translational regulation of Endoglin stability and function is critical for angiogenesis. J. Biol. Chem. 289, 25486-25496. doi: 10.1074/jbc.M114.578609

Park, S. O., Wankhede, M., Lee, Y. J., Choi, E. J., Fliess, N., Choe, S. W., et al. (2009). Real-time imaging of de novo arteriovenous malformation in a mouse model of hereditary hemorrhagic telangiectasia. J. Clin. Invest. 119, 3487-3496. doi: $10.1172 /$ JCI39482 
Peter, M. R., Jerkic, M., Sotov, V., Douda, D. N., Ardelean, D. S., Ghamami, N., et al. (2014). Impaired resolution of inflammation in the Endoglin heterozygous mouse model of chronic colitis. Mediat. Inflamm. 2014:767185. doi: $10.1155 / 2014 / 767185$

Plow, E. F., Meller, J., and Byzova, T. V. (2014). Integrin function in vascular biology: a view from 2013. Curr. Opin. Hematol. 21, 241-247. doi: 10.1097/MOH.0000000000000042

Ray, B. N., Lee, N. Y., How, T., and Blobe, G. C. (2010). ALK5 phosphorylation of the endoglin cytoplasmic domain regulates Smad1/5/8 signaling and endothelial cell migration. Carcinogenesis 31, 435-441. doi: 10.1093/carcin/bgp327

Romero, D., Terzic, A., Conley, B. A., Craft, C. S., Jovanovic, B., Bergan, R. C., etal. (2010). Endoglin phosphorylation by ALK2 contributes to the regulation of prostate cancer cell migration. Carcinogenesis 31, 359-366. doi: 10.1093/carcin/bgp217

Rossi, E., Sanz-Rodriguez, F., Eleno, N., Düwell, A., Blanco, F. J., Langa, C., et al. (2013). Endothelial endoglin is involved in inflammation: role in leukocyte adhesion and transmigration. Blood 121, 403-415. doi: 10.1182/blood-2012-06435347

Santibanez, J. F., Pérez-Gómez, E., Fernandez-L, A., Garrido-Martin, E. M., Carnero, A., Malumbres, M., et al. (2010). The TGF-beta co-receptor endoglin modulates the expression and transforming potential of H-Ras. Carcinogenesis 31, 2145 2154. doi: 10.1093/carcin/bgq199

Shen, F., Degos, V., Chu, P. L., Han, Z., Westbroek, E. M., Choi, E. J., et al. (2014). Endoglin deficiency impairs stroke recovery. Stroke 45, 2101-2106. doi: 10.1161/STROKEAHA.114.005115

Shovlin, C. L. (2010). Hereditary haemorrhagic telangiectasia: pathophysiology, diagnosis and treatment. Blood Rev. 24, 203-219. doi: 10.1016/j.blre.2010.07.001

Takada, Y., Ye, X., and Simon, S. (2007). The integrins. Genome Biol. 8:215. doi: 10.1186/gb-2007-8-5-215
Tian, H., Mythreye, K., Golzio, C., Katsanis, N., and Blobe, G. C. (2012). Endoglin mediates fibronectin/ $\alpha 5 \beta 1$ integrin and TGF- $\beta$ pathway crosstalk in endothelial cells. EMBO J. 31, 3885-3900. doi: 10.1038/emboj.2012.246

Torsney, E., Charlton, R., Parums, D., Collis, M., and Arthur, H. M. (2002). Inducible expression of human endoglin during inflammation and wound healing in vivo. Inflamm. Res. 51, 464-470. doi: 10.1007/PL00012413

van Laake, L. W., van den Driesche, S., Post, S., Feijen, A., Jansen, M. A., Driessens, M. H., et al. (2006). Endoglin has a crucial role in blood cell-mediated vascular repair. Circulation 114, 2288-2297. doi: 10.1161/CIRCULATIONAHA.106.639161

Conflict of Interest Statement: The authors declare that the research was conducted in the absence of any commercial or financial relationships that could be construed as a potential conflict of interest.

Received: 15 October 2014; accepted: 12 December 2014; published online: 07 January 2015.

Citation: Rossi E, Lopez-Novoa JM and Bernabeu C (2015) Endoglin involvement in integrin-mediated cell adhesion as a putative pathogenic mechanism in hereditary hemorrhagic telangiectasia type 1 (HHT1). Front. Genet. 5:457. doi: 10.3389/fgene.2014.00457

This article was submitted to Genetic Disorders, a section of the journal Frontiers in Genetics.

Copyright (C) 2015 Rossi, Lopez-Novoa and Bernabeu. This is an open-access article distributed under the terms of the Creative Commons Attribution License (CC BY). The use, distribution or reproduction in other forums is permitted, provided the original author(s) or licensor are credited and that the original publication in this journal is cited, in accordance with accepted academic practice. No use, distribution or reproduction is permitted which does not comply with these terms. 